\title{
Prevalencia de la enfermedad de Hansen en el barrio Francisco rojas del municipio de Tipitapa
}

\author{
Magdalena Marín Pérez \\ Génesis Carolina Pérez Pilarte \\ dragenesis.dermatologa@gmail.com
}

Recibido: septiembre 2013. Aprobado: septiembre 2016

\section{RESUMEN}

El presente estudio permitió evaluar la prevalencia de la Enfermedad de Hansen en el barrio Francisco Rojas del municipio de Tipitapa, departamento de Managua, durante el período de Diciembre 2012 a Febrero 2013. Para cumplir tal objetivo se realizaron visitas domiciliares a los contactos de los pacientes diagnosticados con Enfermedad de Hansen durante los años 2000 al 2012 en el Centro Nacional de Dermatología, denominados éstos como casos guías. Durante estas visitas se realizó una inspección completa de la piel a todos los contactos, surgiendo así pacientes con lesiones sospechosas de la enfermedad, a los cuales se les realizaron baciloscopía y biopsia para confirmar el diagnóstico y clasificar la enfermedad. Partiendo de los 9 casos guías surgieron 6 casos nuevos. La prevalencia de la enfermedad para el año 2012 en el barrio Francisco Rojas fue de 10.85 por 10,000 habitantes, la tasa de detección fue de 13.02 por 10,000 habitantes y la proporción de casos en menores de 15 años fue $16.66 \%$. Tomando en cuenta estos indicadores se puede concluir que existe un foco activo de lepra en el barrio Francisco Rojas del municipio de Tipitapa, y por sus características epidemiológicas debe considerarse un problema de salud pública. Por lo que se recomienda priorizar el diseño de un programa de atención y vigilancia epidemiológica de la Enfermedad de Hansen para un mejor control en la transmisión de la misma. Palabras clave: Hansen, dermatología, salud pública

\section{SUMMARY}

The present study made it possible to evaluate the prevalence of Hansen's Disease in the Francisco Rojas neighborhood of the municipality of Tipitapa, in the department of Managua, during the period from December 2012 to February 2013. In order to meet this objective, home visits were made to patients' contacts Diagnosed with Hansen's Disease during the years 2000 to 2012 in the National Center of Dermatology, called these as guiding cases. During these visits, a complete skin inspection was performed on all contacts, resulting in patients with suspicious lesions of the disease, who underwent smear microscopy and biopsy to confirm the diagnosis and classify the disease. Starting from the 9 cases, 6 new cases emerged. The prevalence of the disease for the year 2012 in the Francisco Rojas neighborhood was 10.85 per 10,000 inhabitants, the detection rate was 13.02 per 10,000 inhabitants and the proportion of cases in children under 15 years was $16.66 \%$. Taking these indicators into account, it can be concluded that there is an active focus of leprosy in the Francisco Rojas neighborhood of the municipality of Tipitapa, and because of its epidemiological characteristics it must be considered a public health problem. Therefore it is recommended to prioritize the design of a program of care and epidemiological surveillance of Hansen's Disease for a better control in the transmission of the same. Key words: Hansen, dermatology, public health 


\section{INTRODUCCIÓN}

La Organización Mundial de la Salud (OMS) reporta cifras oficiales procedentes de 121 países y territorios, donde la prevalencia mundial de la lepra a principios de 2009 fue de 213,036 casos. Mientras que el número de casos nuevos detectados en 2008 había sido de 249,007, se observaron 35, 971 casos menos, esto atribuido al uso del tratamiento con poliquimioterapia. (OMS, 2010, págs. 5-7)

Para el 2007, en el Centro Nacional de Dermatología, se realizó un estudio en el que se recopiló la información de todos los pacientes diagnósticados con enfermedad de Hansen desde 1980 al 2007. El total de pacientes diagnosticados durante el período mencionado fue de 205. La procedencia fue la siguiente: Managua 107, Chinandega 30, León 16, Masaya 10, RAAS 9, Chontales 8, Carazo 5, Granada 4, Rivas 4, Matagalpa 3, Estelí 1 y Boaco 1. Estos constituyeron los focos leprógenos detectados hasta el 2007. (López, 2007).

Durante el año 2012, se reportaron 8 casos nuevos de Enfermedad de Hansen, de estos el 62.5 $\%$ (5) procedentes del Barrio Francisco Rojas del municipio de Tipitapa, donde el 60\% (3) fueron multibacilares y el $40 \%$ (2) paucibacilares.

Estos datos indican que existe un número significativo de casos de enfermedad de Hansen en este barrio, y con este comportamiento se puede asegurar que de no realizar una intervención a lo inmediato los casos continuaran en ascenso, ya que la mayoría son multibacilares. Es por esta razón que se decidió realizar una búsqueda activa de casos que permita cortar la cadena de transmisión a través del diagnóstico precoz y tratamiento oportuno lo cual conllevaría a prevenir las discapacidades de la población afectada.

\section{MATERIAL Y MÉTODOS}

Tipo de estudio: El estudio es de tipo Observacional, descriptivo, analítico, prospectivo y transversal. En el campo de la investigación clínico-epidemiológica el tipo de estudio se clasifica como estudio de caso.

Área de estudio: El estudio se realizó en el barrio Francisco Rojas del municipio de Tipitapa, departamento de Managua.

Universo y muestra: constituido por todos los pacientes que cumplieron con los criterios de inclusión contemplados, que correspondieron a 28 pacientes.

\section{Criterios de inclusión}


1. Que acepte participar en el estudio

2. Pacientes diagnosticados con Enfermedad de Hansen y registrados en las estadísticas del Centro Nacional de Dermatología a partir del año 2000 al 2012 (Denominados Casos Guías).

3. Contactos familiares de los casos guías.

4. Convivencia intrafamiliar de más de seis meses.

5. Contactos familiares que actualmente no habiten en el núcleo familiar pero que hayan convivido por más de seis meses en el pasado.

6. Contactos no familiares frecuentes (vecinos, amigos)

\section{Procedimiento para recolección de la información}

Se realizaron visitas de campo y se obtuvo la información a través de una encuesta e inspección completa de la piel de los contactos de los pacientes diagnosticados con enfermedad de Hansen registrados en las estadísticas del Centro Nacional de Dermatología a partir del año 2000 al 2012. A los pacientes con lesiones sospechosas de la enfermedad se les realizaron baciloscopía de nariz, lóbulo de la oreja y codos, e histopatología de la lesión para confirmar diagnóstico y clasificar los casos encontrados.

\section{RESULTADOS Y DISCUSIÓN}

En la presente investigación sobre Prevalencia de la Enfermedad de Hansen en el barrio Francisco Rojas, municipio de Tipitapa, departamento de Managua, se realizó una búsqueda activa de casos partiendo de 9 casos guías que corresponden a los pacientes diagnosticados con Enfermedad de Hansen durante el período del 2000 al 2012. Se identificaron 131 contactos, de los cuales el $89.3 \%$ (117) estaban presentes en el momento de las visitas y aceptaron ser examinados. Del total de personas examinadas, el 16.2 \% (19) se clasificaron como casos sospechosos de la Enfermedad de Hansen, de los cuales se confirmaron 6 casos.

En relación a la edad de los pacientes estudiados, se observa que, en los casos nuevos el 95\% de los afectados se encuentran en el rango de edad de 20 a 60 años, estos datos se corresponden con los encontrados en el estudio realizado por López (2007) sobre el Comportamiento Epidemiológico y Social de los casos de lepra en el Centro Nacional de Dermatología 1980 - 2007 que reporta que el 
grupo de edad más afectado fueron los mayores de 15 años con el $81.95 \%$. Igualmente corresponde con lo reportado por Jean L. Bolognia donde los grupos de edades más afectados son los de 10 a 15 años y los de 30 a 60 años. (Figura 1)

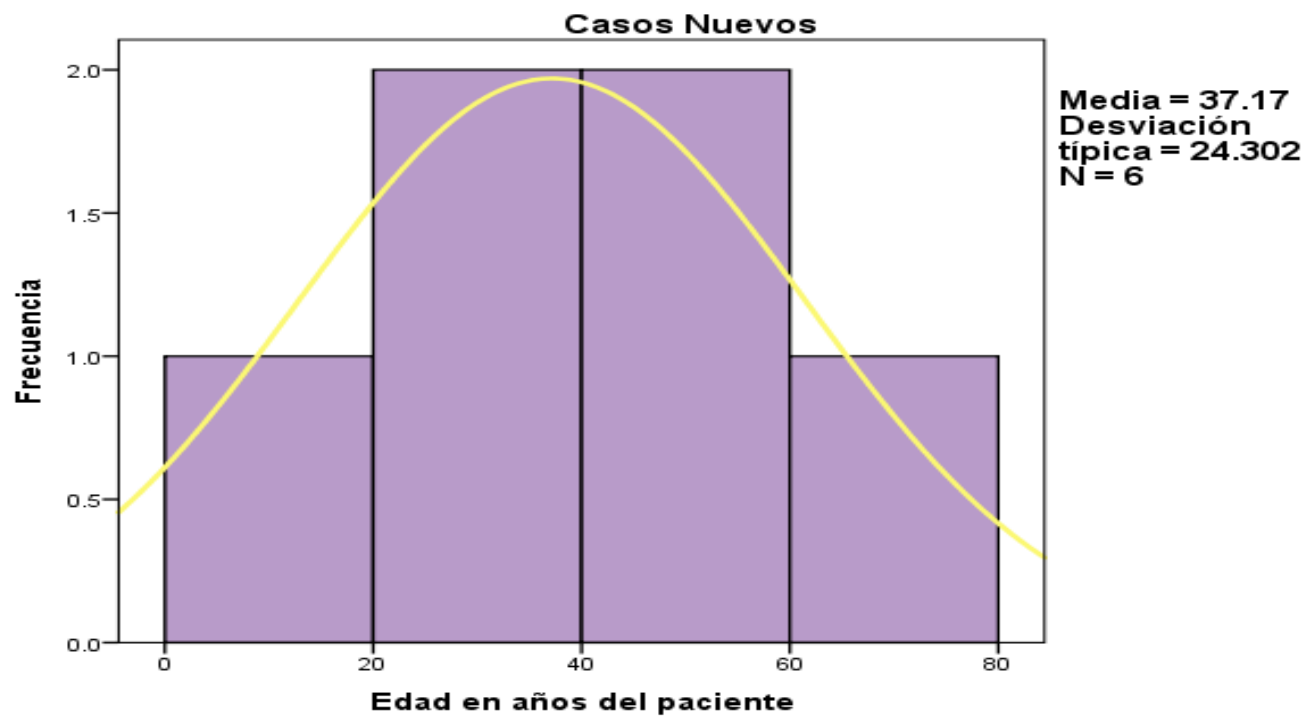

Figura 1. Edad en años del paciente

Con respecto al sexo se observa que en los casos nuevos, predominó el sexo masculino con una razón hombre: mujer 2:1, lo que coincide con lo encontrado en la mayoría de los estudios de lepra y particularmente en el realizado por López, (2007) sobre el Comportamiento Epidemiológico y Social de los casos de lepra en el Centro Nacional de Dermatología 1980- 2007 que reporta que el sexo masculino fue el más afectado con el 60.97 \%. (Figura 2)

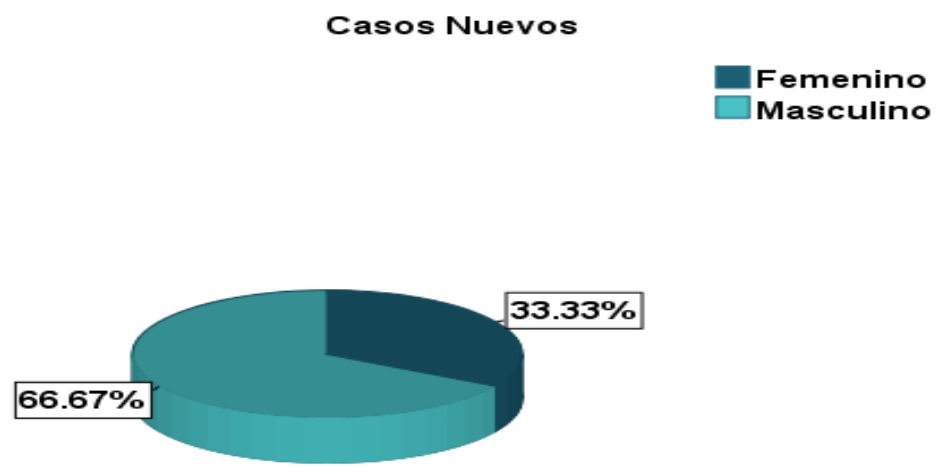

Figura 2. Sexo de los casos nuevos 
Al razonar las formas clínicas de la enfermedad de Hansen en los casos nuevos, se observa que al agrupar los casos dimorfos y los indeterminados constituyen el $66.66 \%$, lo que traduce que existe una transmisión activa de la enfermedad, y que si estos pacientes no son tratados oportunamente pueden evolucionar hacia un polo Lepromatoso, y por lo tanto Multibacilar, lo que constituye un mayor riesgo de transmisibilidad y secuelas para el paciente. (Figura 3)

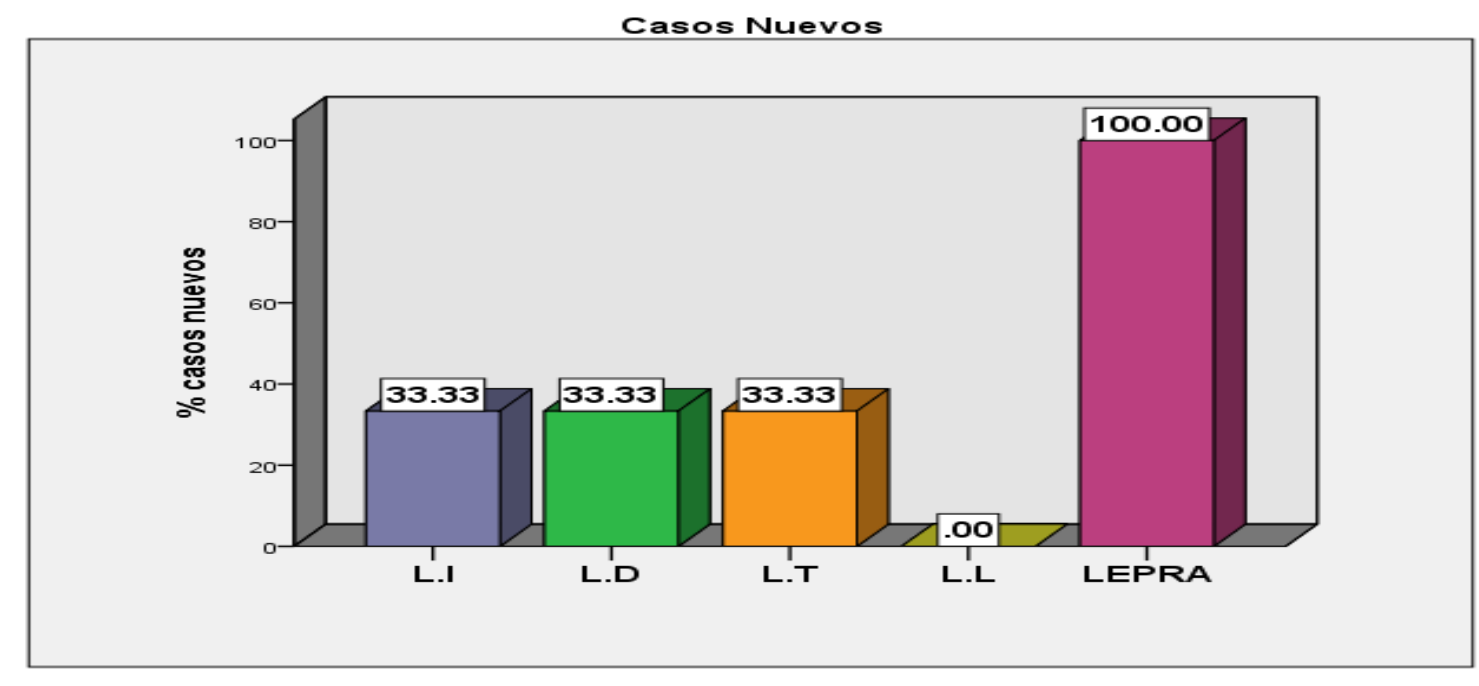

Figura 3. Formas clínicas enfermedad de Hansen casos nuevos

La prevalencia de la Enfermedad de Hansen para el año 2012 en el barrio Francisco Rojas fue de 10.85 por 10,000 habitantes. Según lo reportado por la ILEP en el 2001, la prevalencia teóricamente mide la morbilidad de la enfermedad, pero no mide la transmisión de ésta, por lo tanto, se calculó la tasa de detección que fue de 13.02 por 10,000 habitantes, indicador más útil para estimar la transmisión de la enfermedad en un área, siendo esta la tasa que más se aproxima a la tasa de incidencia. La proporción de casos en menores de 15 años fue $16.66 \%$. Tomando en cuenta este indicador podemos decir que hay una transmisión activa y reciente de la enfermedad, dado que la ILEPmenciona quecuando la afectación se da en niños, estos deben haber sido infectados recientemente.

Se relacionó las condiciones de vida con la presencia o no de enfermedad de Hansen, mediante la prueba estadística de Phi y Kramer, donde resultó que en este estudio ambas variables son independientes, es decir no existe asociación estadística entre las variables. Se considera que este resultado se debe a que las condiciones de vida no son el único factor que influye para padecer la enfermedad, sino que influyen además otros factores tales como la predisposición genética, factores 
nutricionales, factores inmunológicos, carga bacilar de los contactos y factores dependientes del bacilo. (Figura 4)

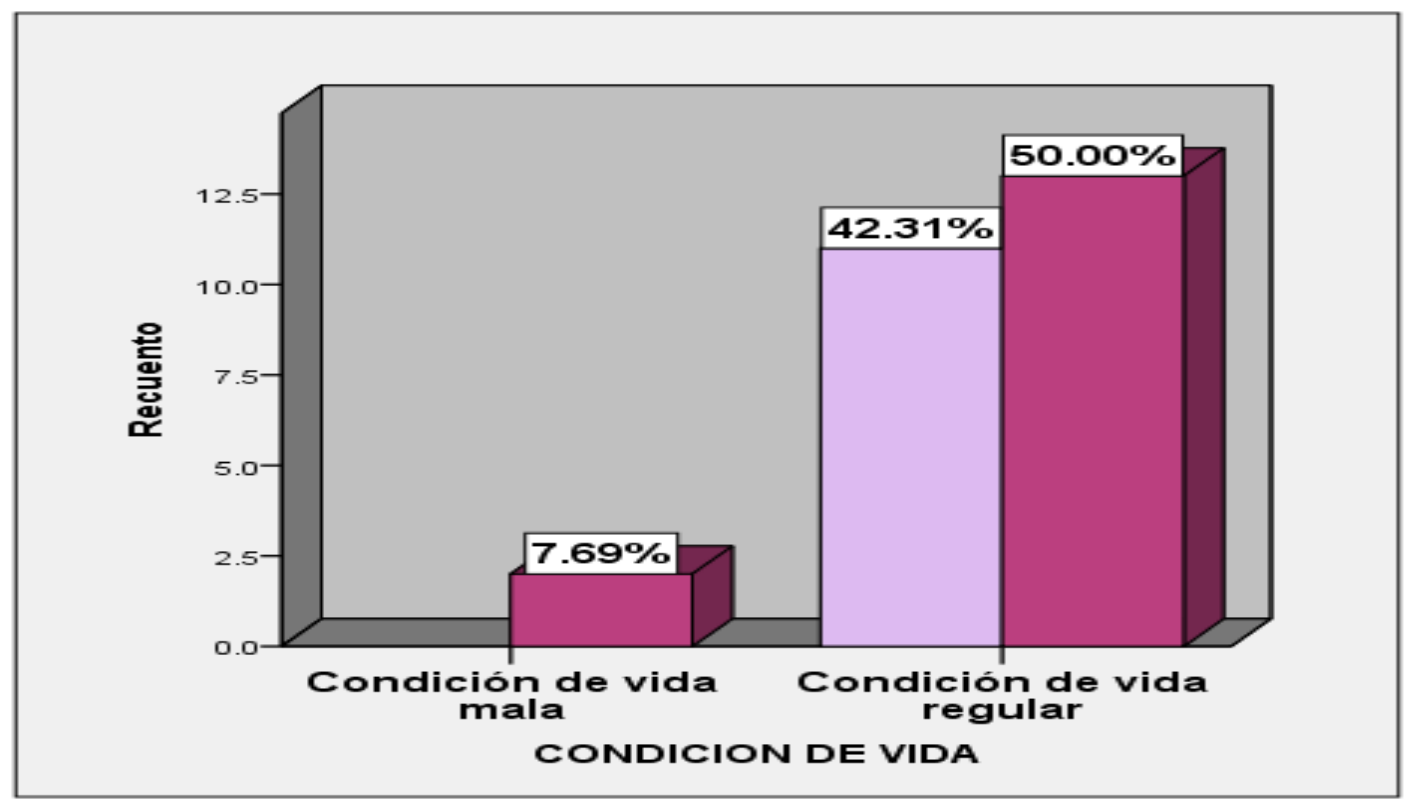

Figura 4. Relación condiciones de vida y enfermedad de Hansen

\section{CONCLUSIONES}

Existe uniformidad en las características sociodemográficas y condiciones de vida de los pacientes estudiados.

Existe un proceso activo de transmisión de la enfermedad, demostrado por el predominio en los casos nuevos de la forma dimorfa e indeterminada, afectación a menores de 15 años (16.6 \%) y tasa de detección de 13.02.

En el barrio Francisco Rojas del municipio de Tipitapa existe un foco activo de lepra, y por sus características epidemiológicas debe considerarse un problema de salud pública.

No existe relación entre las condiciones de vida y la enfermedad de Hansen, ya que deben considerarse otros factores que influyen en el contagio de la enfermedad

\section{RECOMENDACIONES}

Priorizar el diseño de un programa de atención y vigilancia epidemiológica de la Enfermedad de Hansen en el Ministerio de Salud que involucre a la comunidad. 
Descentralizar el tratamiento desde el Centro Nacional de Dermatología al Hospital Yolanda Mayorga, ya que existe personal capacitado (Dermatóloga) que puede dar seguimiento a los casos. Además esto permitiría garantizar el cumplimiento del tratamiento por parte de los pacientes debido a las dificultades económicas que éstos poseen.

Dar a conocer los resultados del presente estudio a las autoridades de los diferentes niveles del Ministerio de salud, así como a los organismos involucrados en el tema de la Enfermedad de Hansen.

Realizar futuras investigaciones en los lugares donde hay focos de lepra, que evalúen además de los aspectos clínicos y epidemiológicos, el conocimiento que tienen sobre la enfermedad de Hansen tanto trabajadores de la salud como la población general.

\section{REFERENCIAS}

López, S. (2007). Comportamiento Epidemiológico y social de los casos de lepra en el Centro Nacional de Dermatología 1980 - 2007. Managua.

OPS. (2006-2010). Estrategia mundial para aliviar la carga de la lepra y sostener las actividades de control de la enfermedad. Recuperado el Jueves 13 de Febrero de 2013http://www.who.int/mediacentre/factsheets/fs101/es/index.html. 